\title{
NUMERICAL SIMULATION OF AIR-WATER BUBBLY FLOW IN AXIAL FLOW PUMP IMPELLER
}

\author{
Minguan Yang, Xiaolian Wu ${ }^{*}$, Can Kang \\ School of Energy and Power Engineering, Jiangsu University; Zhenjiang, Jiangsu \\ Province,P.R China 212013 \\ * Corresponding author, Address: School of Energy and Power Engineering, Jiangsu \\ University; *Zhenjiang, Jiangsu Province,P.R China ,Tel: 13775376332, Fax: 0511-8878 \\ 0216,Email:wuxiaolian0362@163.com
}

Abstract: In this paper, the lift-law method was applied to design the axial flow pump. Based on Reynolds time-averaged N-S equations and standard $k-\varepsilon$ turbulent model, three-dimensional air-water bubbly flow in an axial flow pump impeller was simulated. Under the condition of bubble void fraction of $5 \%$, pressure and void fraction distributions were respectively obtained, movement of bubbles in the impeller channel was analyzed. The numerical results demonstrate that air bubbles accumulate at the blade back near the impeller inlet because the lowest pressure on blade back happens here.

Keywords: axial flow pump, impeller, numerical simulation, air-water bubbly flow

\section{INTRODUCTION}

Axial flow pump possesses the characteristics of large flow rate, low head and simple structure, and has been widely used in agricultural and industrial engineering fields as an important transporting device. During the last decade, there has been a growing interest in pumping two-phase mixtures. Usually, the appearance of gas in the liquid leads to a strong decrease in efficiency. With respect to the development of turbo-machines with improved performance in the multiphase flow region, it is important to

Please use the following format when citing this chapter:

Yang, M., Wu, X. and Kang, C., 2009, in IFIP International Federation for Information Processing, Volume 293, Computer and Computing Technologies in Agriculture II, Volume 1, eds. D. Li, Z. Chunjiang, (Boston: Springer), pp. 545-552. 
acknowledge information of each phase's parameter distributions inside the pump (H.-M.Prasser et al ,2003). Relevant studies of internal flow with computational fluid dynamics (CFD) are mainly focused on pure water flow conditions. As for multiphase flows in axial flow pump impeller, especially the air-water flow involving bubble-flow interaction, few researches are reported. So the present work focuses on visualizing the gas fraction distribution inside the rotating impeller of an axial flow pump delivering two-phase fluid.

Under the condition of bubble void fraction of 5\%, this paper adopts commercial CFD software FLUENT to simulate the air-water bubbly flow in an axial flow pump impeller.

\section{NUMERICAL SIMULATION}

\subsection{Computation model}

The designed axial flow impeller has 4 blades, outlet diameter of $200 \mathrm{~mm}$ and hub diameter of $104 \mathrm{~mm}$. The pump operates at flow rate of $360 \mathrm{~m}^{3} / \mathrm{h}$ and rotation speed is of $1450 \mathrm{r} / \mathrm{min}$ (See Fig. 1).

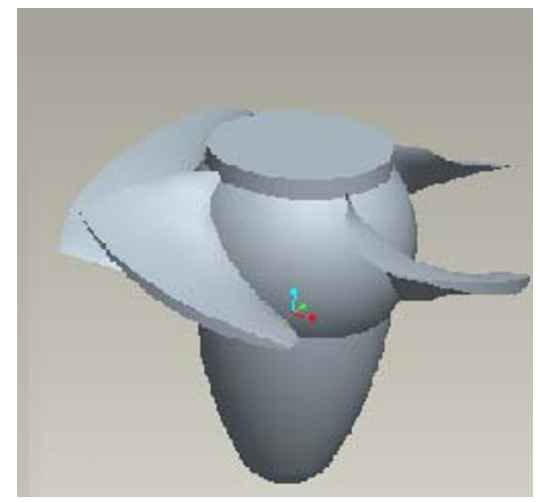

Fig.1.Three-dimensional model

\subsection{Governing equations}

As the pump impeller rotates at uniform angular speed, a necessarily established rotating Cartesian coordinate system rotates synchronously with impeller and coincides with impeller's axis. The convection terms of control equations are discreted with a second order upwind scheme, while the source term and diffusion term are handled with second order central differential scheme(YU Zhi-Yi et al,2007) .Based on Reynolds time-averaged N-S 
equations, with hypothesis of Bossinesq eddy viscosity coefficient (Falcimaigne $\mathrm{J}$ et al, 2005), the basic equations to be solved contain the continuity equation

$$
\begin{aligned}
& \frac{\partial}{\partial_{t}}\left(\alpha_{l} \rho_{l}\right)+\frac{\partial}{\partial_{x_{i}}}\left(\alpha_{l} \rho_{l} u_{l j}\right)=0 \\
& \frac{\partial}{\partial_{t}}\left(\alpha_{g} \rho_{g}\right)+\frac{\partial}{\partial_{x_{i}}}\left(\alpha_{g} \rho_{g} u_{g j}\right)=0
\end{aligned}
$$

and the momentum equations

$$
\begin{aligned}
& \frac{\partial}{\partial_{t}}\left(\alpha_{g} \rho_{g} u_{g i}\right)+\frac{\partial}{\partial_{x_{j}}}\left(\alpha_{g} \rho_{g} u_{g i} u_{g j}\right)= \\
& -\alpha_{g} \frac{\partial p}{\partial x_{i}}+\alpha_{g} \rho_{g} f_{g i}+M_{g i} \\
& \frac{\partial}{\partial_{t}}\left(\alpha_{l} \rho_{l} u_{l i}\right)+\frac{\partial}{\partial_{x_{j}}}\left(\alpha_{l} \rho_{l} u_{l i} u_{l j}\right)=-\alpha_{l} \frac{\partial p}{\partial x_{i}} \\
& +\frac{\partial}{\partial x_{j}}\left[\alpha_{l} \mu_{t}\left(\frac{\partial u_{l i}}{\partial x_{j}}+\frac{\partial u_{l j}}{\partial x_{i}}\right)\right]+\alpha_{l} \rho_{l} f_{l i}+M_{l i}
\end{aligned}
$$

where subscript $g$ and $l$ represent gas phase and liquid phase respectively.

\subsection{Turbulent models}

With the $k-\varepsilon$ turbulent model, the equations has the form the $k$ equation:

$$
\begin{aligned}
& \rho \frac{\partial\left(\alpha_{l} \rho_{l} k\right)}{\partial t}+\frac{\partial\left(\alpha_{l} \rho_{l} u_{l j} k\right)}{\partial x_{j}}=\frac{\partial}{\partial x_{j}}\left[\alpha_{l} \frac{\mu_{t}}{\sigma_{k}} \frac{\partial k}{\partial x_{j}}\right] \\
& +\alpha_{l} \mu_{t} \frac{\partial u_{i}}{\partial x_{j}}\left(\frac{\partial u_{j}}{\partial x_{i}}+\frac{\partial u_{i}}{\partial x_{j}}\right)-\alpha_{l} \rho_{l} \varepsilon
\end{aligned}
$$

the $\varepsilon$ equation: 


$$
\begin{aligned}
& \frac{\partial\left(\alpha_{l} \rho_{l} \varepsilon\right)}{\partial t}+\frac{\partial \alpha_{l} \rho_{l} u_{l j} \varepsilon}{\partial x_{j}}=\frac{\partial}{\partial x_{j}}\left[\alpha_{l} \frac{\mu_{t}}{\sigma_{\varepsilon}} \frac{\partial \varepsilon}{\partial x_{j}}\right] \\
& +\alpha_{l} \mu_{t} \frac{c_{1} \varepsilon}{k} \frac{\partial u_{j}}{\partial x_{j}}\left(\frac{\partial u_{i}}{\partial x_{j}}+\frac{\partial u_{j}}{\partial x_{i}}\right)-c_{2} \alpha_{l} \rho_{l} \frac{\varepsilon^{2}}{k}
\end{aligned}
$$

Where $\sigma_{k}$ and $\sigma_{\varepsilon}$ are Prandtl numbers of $k$ and $\varepsilon$ respectively, of which $\sigma_{k}=1.0, \sigma_{\varepsilon}=1.3$. And other parameters are as follows: $C_{\mu}=0.09$, $C_{1}=1.44, C_{2}=1.92$.

\section{BOUNDARY CONDITIONS}

According to the flow characteristics at the inlet of impeller, law of mass conservation and hypothesis of irrotationality, inlet axial velocity is determined. Tangential velocity and radial velocity are set to zero. Turbulent kinetic energy at the inlet is $1 \%$ of average kinetic energy of the bulk flow. And its dissipation rate is deduced from turbulent kinetic energy and characteristic length of inlet, that is

$$
\varepsilon_{i n}=\frac{c_{\mu}^{3 / 4} k_{i n}^{3 / 2}}{k y_{m}}
$$

Where $y_{m}$ is distance between calculation point and the wall.

At the outflow boundary of the computational region, velocity, turbulent kinetic energy and its dissipation rate are defined with Neuman boundary condition.

$$
\frac{\partial \phi}{\partial n}=0(\phi=u, v, w, k, \varepsilon)
$$

At solid walls, Neuman boundary condition is used in gas fraction, and the no-slip boundary condition is applied to air-water two-phase velocities $(u 、 v 、 w=0)$. Near the wall, the wall function method is employed, the distance between point $\mathrm{P}$ and the wall is denoted by $y_{P}$, so that velocity of point $\mathrm{P} u_{P}$, turbulent kinetic energy $k_{P}$ and its dissipation rate are determined by (Launder B E et al, 1974).

$$
\frac{u_{p}}{u_{\tau}}=\frac{1}{\kappa} \ln \left(E y_{p}^{+}\right)
$$


Impeller

$$
\begin{aligned}
& k_{p}=\frac{u_{\tau}^{2}}{\sqrt{C_{\mu}}} \\
& \varepsilon_{p}=\frac{u_{\tau}^{3}}{\kappa y_{p}}
\end{aligned}
$$

Where $y_{p}^{+}=\frac{\rho u_{\tau} y_{p}}{\mu}=\frac{\rho c_{\mu}^{1 / 4} k_{p}^{1 / 4} y_{p}}{\mu}, \quad$ skin friction coefficient $u_{\tau}=\sqrt{\tau_{w} / \rho} . \quad \mathrm{E}$ and $\mathrm{\kappa}$ are constant and defined as 9.011 and 0.419 respectively .

\section{RESULTS}

\subsection{Static pressure distributions}
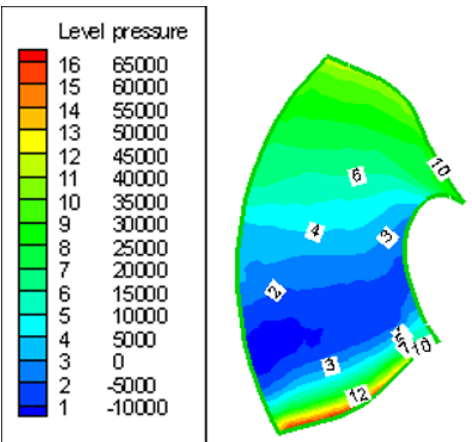

Fig.2.static pressure distribution on blade back of the impeller
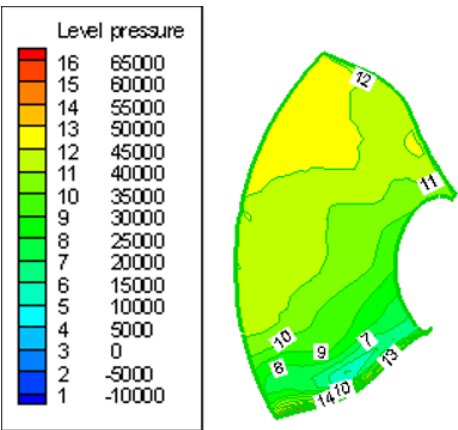

Fig.3. static pressure distribution on blade front of the impeller 
As shown in Fig.2, pressure contours on blade back progress in radial direction and get intensive approaching the leading edge. It is found that an obvious low-pressure area exists at the blade tip near the impeller inlet.

Except the leading edge, pressure contours on blade front are sparse in Fig.3. Pressure variation mainly occurs in radial direction and pressure increases from impeller hub to blade tip.

With comparison of Fig.2 and Fig.3, it can be found that static pressure on blade front is higher than that on corresponding position of blade back.

\subsection{Relative velocity distributions}

According to static pressure distributions of the impeller channel, we can analysis the relative velocity distributions of the blade back and front. Based on Bernoulie's Equation

$$
\frac{p}{\rho g}+\frac{w^{2}}{2 g}=\text { const }
$$

The $w$ difference between blade back and blade front exhibits same variation tendency with pressure difference. Assuming uniform pressure distribution at the inlet flow section, then $w$ at two blade sides at the inlet are of the same. As shown in Fig.2 and Fig.3, static pressure increases gradually from blade inlet to outlet, so the velocity $w$ decreases. And it is obvious that $w$ on blade back is higher than that on corresponding position of blade front.

\subsection{Gas fraction distributions}

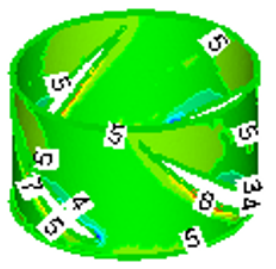

(a)Cylindrical flow surface $1\left(r_{1}=60 \mathrm{~mm}\right)$

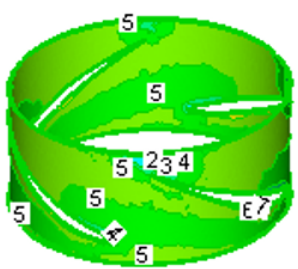

(b) Cylindrical flow surface $2\left(r_{2}=80 \mathrm{~mm}\right)$ 
Impeller

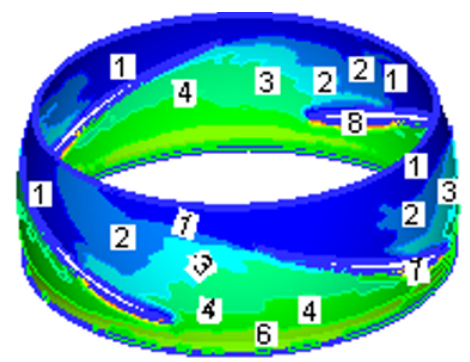

(c) Cylindrical flow surface $3\left(r_{3}=100 \mathrm{~mm}\right)$

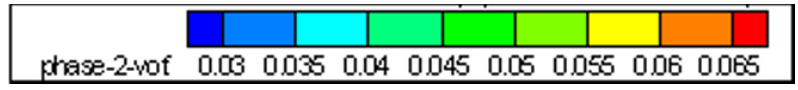

Fig.4.Gas fraction distributions inside the impeller of the axial pump

From Fig.4, we can find that accumulation of air volume happens closely behind the leading edge of the blade back. Along the blades the accumulation became weaker. Along the same axial direction, with the increase of radius, gas fraction decreased. And total gas fraction on blade back is higher than that on blade front. That is decided by the lower pressure regions on blade back. And under action of pressure difference and high speed rotation, fluid flows through flange clearance from blade front to back, air bubbles accumulate here.

\section{CONCLUSION}

(1) Commercial CFD technology was successfully applied to simulate the void fraction distributions inside the impeller of an axial flow pump transporting multiphase medium. The results can reflect the flow patterns in the impeller.

(2) Simulation was carried out at a volumetric air flow rate fraction $\beta$ at the pump inlet of $5 \%$, a clearly visible accumulation of air was observed at the blade back near the impeller inlet, because the lowest pressure on blade back happens here.

(3) These results provide useful reference for optimization design of axial flow impeller.

\section{ACKNOWLEDGEMENTS}

The work presented was supported by a funding of nation of science. 


\section{REFERENCES}

Falcimaigne J,Brac J, Charron Y, et al. Multiphase Pumping: achievement and Perspectives [J]. Oil \& Gas Science and Technology, Rev. IEP, 2005,57(1): 99-107.

H.-M.Prasser,D.Baldauf,J.Fietz,U.Hampel. Time resolving gamma-tomography for periodically changing gas fraction fields and its application to an axial pump [J]. Flow Measurement and Instrumentation, 2003(14):119-125.

HUANG Si, WU Yu-lin. 3-D Calculation of gas-oil flow in rotodynamic pump based on a bubbly flow model, SHUILI XUEBAO, 2001(6): 57-61.

Launder B E, Spalding D B. The numerical computation of turbulent flows. Compute Methods Appl. Mech.Eng., 1974(3)269-306.

LI Long, WANG Ze. Numerical Simulation of Interior Flow Field in Axial Pump. FLUID MACHINERY,2007(35): 16-18

Lu Jinling, Review of Research on Gas-liquid Two-phase flow Pumps, FLUID MACHINERY 29(2001): 12-15.

YU Zhi-Yi, Gao Shu-Liang, WANG Guo-Yu. CFD Analysis of The Air-water Bubbly Flow in a Multiphase Rotodynamic Pump Impeller [J]. JOURNAL OF ENGINEERING THERMOPHYSICS, 2007 (28) :46-48.

ZHENG Wei, YANG Jun-hu, MA Lei. Numerical Simulation of 3D Turbulent Flow Through the Full Passage of Submersible Axial Flow Pump, Journal of Gansu Science, 2007(19): 105-107. 